\title{
Decision Theory, Symmetry and Causal Structure: Reply to Measham and Weisberg
}

\author{
MICHAEL CLARK AND NICHOLAS SHACKEL
}

\section{Best paradoxical cases}

It is of the nature of the two-envelope paradox when fully developed that not only are there several plausible ways of evaluating swapping, but that there are intricate semi-dependencies between those ways. Consequently a certain amount of complexity has to be marshalled in order to be sure of both covering the full extent of the problem and eliminating those parts which are inessential or not paradoxical.

Finite two-envelope cases are ones in which there is an upper bound on the amount of money in an envelope. Everybody agrees that the finite cases are solved by the considerations we give in section 1 of our paper.

The expectations in infinite two-envelope cases are identical to infinite sums, which mathematicians call 'series'. So, to speak of an expectation, such as $E(B-A)$, in these cases, is to speak of a series. Series are identified by the infinite sequence of their terms; rearranging the terms of a series gives you a different series. The sum of a series is defined to be the limit of the sequence of its partial sums. So when we speak of the behaviour of expectations in the infinite cases we are speaking of the behaviour of series, which is defined in terms of the behaviour of sequences. These are among the fruits of the nineteenth century demand for rigour in that part of mathematics called 'analysis'. 
In our Appendix (pp. $430 \mathrm{ff}^{1}{ }^{1}$ ) we make use of those fruits in analysing the infinite two-envelope cases. We consider 'five different series which may be held to give the average expected gain on swapping' (p. 430). On the basis of showing how they depend on the behaviour of a certain family of sequences, $\sqrt{ } 2^{n} p_{n} \cdot{ }^{2}$ and on the senses in which a series can be said to be well defined, we categorize the kinds of infinite cases. We show which kinds of infinite case are not paradoxical (p. 435, remarks 1 and 2), and the conditions under which certain ways of evaluating swapping can be ignored because they fail to give even a semblance of an answer (p. 435, remarks (a), (b) and 3). We identify the two kinds of paradoxical cases, only one of which had been previously identified and discussed as such in the literature (p. 435, remarks 4 and 5).

The kind previously identified were cases in which the average expected gain on swapping, calculated as $E(E(B \mid A)-A)$, is infinite. We named such cases 'unbounded paradoxical' and pointed out that divergence to infinity is only controversially a way for an infinite sum to be well defined. Some earlier discussions claimed that the paradox was solved by rejecting divergent expectations. That is to say, the paradox as stated initially gets you to calculate an expectation based on a supposition of what is in your envelope, $E(B \mid A)-A$, which when you find it to be positive persuades you to swap. But, say those who reject divergent expectations, a condition on the propriety of that

\footnotetext{
${ }^{1}$ Page references are to Clark and Shackel 2000 unless otherwise indicated.

${ }^{2}$ Mathematicians tend to use curly brackets both for sets and for naming sequences: here it would be ' $\left\{2^{n} p_{n}\right\}$ '. Since angle brackets are standardly understood by philosophers to apply to ordered sets, we have specified sequences and families of sequences by their use.
} 
procedure is that the average such expected gain, $E(E(B \mid A)-A)$, must be well defined, and since divergence is not a way to be well defined, and $E(E(B \mid A)-$ A) diverges to infinity, the paradox is solved.

The second kind of paradoxical case, which we called 'best paradoxical', are ones in which the average expected gain on swapping, calculated as $E(E(B \mid A)-A)$, is finite. Such cases cannot be solved by the rejection of divergence. Since we exhibited such a case, the question of whether divergence is improper is moot so far as solving the two-envelope paradox in general goes. We shall therefore assume that Measham and Weisberg are concerned only with best paradoxical cases.

\section{Decision theory}

We are puzzled by many things Measham and Weisberg say about decision theory.

They seem to be confused about the existence conditions for the expectations with which we are concerned. On page 000 (Measham and Weisberg 2003) they say

If there are an infinite number of possibilities over which to sum, the $E U$ [expected utility] is the value to which the series absolutely converges; otherwise it is undefined.

This statement is false. Absolute convergence ${ }^{3}$ is a stronger condition than convergence, and all that is needed for the $E U$ of an infinite number of possibilities to be defined is for it to converge. These conditions can come

\footnotetext{
${ }^{3} \Sigma U_{n}$ converges absolutely means that $\Sigma\left|U_{n}\right|$ converges.
} 
apart for alternating series (ones in which the signs of the terms alternate), many of which converge but do not converge absolutely.

In footnote 2 they say

If a sum $\Sigma U_{n}$ absolutely converges to $s$, then any $\operatorname{sum} \Sigma V_{n}$, obtained by rearranging terms of $\Sigma U_{n}$, will also converge to $s$.

This too is false. It could be made true if 'also converge to s' is changed to 'also converge absolutely to s', but we think it is probably a misstatement of the rearrangement theorem:

If a sum $\Sigma U_{n}$ absolutely converges, and $\Sigma U_{n}$ converges to $s$, then any $\operatorname{sum} \Sigma V_{n}$, obtained by rearranging terms of $\Sigma U_{n}$, will also converge to $s$.

The point is that what a series converges to, and converges absolutely to, need not be the same. For example, when $E(B-A)$ is absolutely convergent, $E(B-A)=0$, but the value to which $E(B-A)$ absolutely converges, $\Sigma\left|d_{n}\right|$, is strictly positive (since at least one term is positive). ${ }^{4}$

These misunderstandings vitiate what they say about $E(B-A)$ and, we think, lead them to misunderstand the role of our lemma on p. 433-which states that $E(A)$ is finite iff $E(B-A)$ is absolutely convergent-in analysing the kinds of paradoxical case there are. They say:

Thus the $E U$ of swapping would be the expectation of $B-A$, $E(B-A)$, except that this sum does not converge absolutely; given appropriate rearrangements, the sum can be made to converge to any value. So decision theory does not rank

\footnotetext{
${ }^{4}$ The sequence $V_{d_{n}}$. is defined on p. 431 .
} 
swapping against sticking. Clark and Shackel give this result surprisingly little consideration. (Measham and Weisberg 2003, p. 000)

There are two claims here, the second dependent on the first. The first is that decision theory can evaluate swapping only by use of $E(B-A)$, and the second that if $E(B-A)$ doesn't converge absolutely then decision theory makes no recommendation. Even if we granted the first claim, the short answer is that this result is no result. It is quite possible for $E(B-A)$ not to converge absolutely, yet to converge to 0 , when decision theory will say be indifferent to swapping. We mention this possibility in remark (i) on p. 434, and give there an example in which $E(\mathrm{~A})$ is not finite, so $E(B-A)$ does not converge absolutely (by the lemma), but converges to 0 (because the sequence $\sqrt{ } 2^{n} p_{n}$, is null). In such cases every suggested way of calculating choiceworthiness ${ }^{5}$ but $E(A)-E(B)$ is identically zero, so no paradox arises.

From now on, we will assume they said what they ought to have said about convergence. Then their second claim, combined with the above quotation similarly corrected, becomes the claim that if $E(B-A)$ doesn't converge then it is undefined and so decision theory makes no recommendation. Now their criticism of us for giving 'this result surprisingly little consideration' seems merely odd, since if that really were the full solution to the paradox it's a solution we have given and upon which they are relying. For we say, as they quote us saying, that expectations for which the corresponding series has no defined sum are themselves undefined and need not be considered. Their

${ }^{5}$ See pp. $431-32$. 
criticism can only amount to pointing out that having solved it thus, we have then deluded ourselves that there was some further problem to be solved.

But of course, the reason we give it little consideration is because we reject their first claim, that decision theory can evaluate swapping only by use of $E(B-A)$. This takes us on to their next criticism. The further problem with which we have deluded ourselves would be that three other expectations whose infinite sums do converge absolutely [for the reasons given, strike 'absolutely']: $E(E(B \mid A)-$ $A), E(B-E(A \mid B))$ and $E(E(B-A \mid A+B)) \ldots$ are not expressions for the $E U$ of swapping given the standard definition of $E U$. Instead of each term in the series corresponding to a possible outcome, these series have as terms the sum of the utility and probability of two possible outcomes. By employing these average expectations, Clark and Shackel go beyond standard decision theory, and become responsible for the formulation and justification of a new theory in which average expectations play a role. (Measham and Weisberg 2003, p. 000)

We can make little sense of this claim. Perhaps the first thing we should say is that if we have deluded ourselves by thinking this was some further problem, it is a delusion we seem to share with most other authors in the literature. Since expectations such as $E(E(B \mid A)-A)$ are what those authors have been thinking about, we could hardly just ignore these contenders without ignoring previous discussions of the paradox. 
We don't understand what criteria they are applying to restrict definitions of expected utilities in order to exclude our three contenders for evaluating swapping.

in the central heartland of decision theory ... the simple Bayesian rule holds good: desirability measures choiceworthiness. (Jeffrey 1990, p. 20)

What Jeffrey means by desirability is what is sometimes referred to as utility and sometimes as value. In our paper we used 'value', our critics use 'utility'. But whichever term is used, standard decision theory simply imports probability theory wholesale, and defines the value of an act to be the expectation, conditional on that act, of a random variable which represents the value of outcomes numerically. Expectations are defined within probability theory. We relied on a number of standard theorems and definitions of probability theory, among which are:

If $X$ and $Y$ are random variables, $n$ and $m$ real numbers, then

(a) $n X+m Y+k$ is a random variable

(b) $E(n X+m Y+k)$ is an expectation

(c) $E(X \mid Y)$ is a random variable (Feller 1968, p. 223)

(d) $E(E(X \mid Y))$ is an expectation.

When, as in the raw paradox, you suppose $x$ to be in your envelope and calculate 5/4 $x$ as the expectation for the other, what you are actually calculating is a random variable, $E(B \mid A)-A$, and so you must calculate the 
expectation of that random variable $E(E(B \mid A)-A)$ to get properly started. ${ }^{6}$ As is evident from our paper (and many earlier ones), that point on its own solves all the finite cases.

Our three contenders are therefore proper expectations. It isn't true that $E(B-A)$ is the only way decision theory offers to evaluate swapping, and, exciting as it would be to be able to claim otherwise, nothing we have done goes beyond standard decision theory.

\section{Symmetry}

What Measham and Weisberg are proposing to solve the paradoxes amounts to rejecting contenders for evaluating swapping if they are based on conditionalization. We need to see some principled reasons for doing so, not just a rejection because they get the wrong result (for if that was all that was needed, we could all have saved ourselves a great deal of time!). We think it unlikely that there are principled reasons to ignore these contenders, since it is an absolutely standard technique to compute expectations by conditioning (see

${ }^{6}$ One terminological point can now be explained: although we used the phrase 'average expected gain on swapping' to distinguish references to, for example, $E(E(B \mid A)-A)$, from references to, for example, $E(B \mid A)-A$, it would have been quite proper to have called them expected gains on swapping, since that is what they are. We did this because random variables such as $E(B \mid A)-A$ have been referred to as expectations in the earlier literature due to a notational ambiguity. For example, if $c$ is a constant, then while $E(B \mid A)-A$ is a random variable, $E(B \mid A=c)-c$ is an expectation which is sometimes misleadingly written as $E(B \mid A=c)-A$ and even just as $E(B \mid A)-A$. Perhaps this practice should be deprecated, but since mathematicians seem to negotiate the ambiguity without problem, it is unlikely to change. 
for example Ross 1988, pp. 285 ff.). Most of the time it doesn't matter which way you condition (see p. 436), but sometimes it does, and the broader question which the two-envelope paradox raises, in particular the new variety we discovered and named 'best paradoxes', is how you get it right when it matters which way you condition.

We think dismissing the best paradoxes because in these cases $E(B-A)$ is undefined $^{7}$ is a way of ignoring the problem rather than dealing with it. One could take the same approach to Newcomb's problem on the grounds that cases in which the evidential structure and causal structure get out of kilter with each other are cases in which decision theory should simply be given up. But some have thought that the right approach is to modify standard decision theory so that in taking into account the evidence it does so in a manner which is constrained by the causal structure. We think something similar applies here.

Compare the standard case with the variant we describe on p. 426:

Determine $A$ according to the probability distribution ... : $p_{0}=1 / 12, p_{n}=1 / 2 p_{n-1}+1 / 2(1 / 4)^{n}$ for $n \geq 1 .{ }^{8}$ Let the probability of $A$ $=1$ be $p_{0}$ and the probability of $A=2^{n}, n \geq 1$, be $p_{n-1}+p_{n}$. Then

\footnotetext{
${ }^{7}$ Strongly in these cases, since the failure of convergence is not divergence to infinity but divergence by oscillation.

${ }^{8}$ The distribution was introduced and correctly described on p. 423, but erroneously re-described on p. 426, and we take the opportunity to correct it here: we had $p_{n+1}=1 / 2 p_{n} \ldots$ in place of $p_{n}=1 / 2 p_{n-1} \ldots$
} 
determine $B$ thus: if $A=1, B=2$, else $B=$ half or double $A$, with the probability ratios $p_{n-1}: p_{n}$. If $A$ comes out as 4 , for example, the expectation for B is $\left(2 p_{1}+8 p_{2}\right) /\left(p_{1}+p_{2}\right)=4 \%$. You are handed the first sealed envelope containing the sum $A$ and given the option of swapping it for the second, whose content is $B$. It will always be rational to swap and the average expected gain will be $7 / 12$, though the expectations for each envelope have no finite mean.

In both the standard case and the two-stage variant described above, $E(B-A)$ is undefined, so according to Measham and Weisberg decision theory has no recommendation to make. $E(E(B-A \mid A+B))$ gets the right answer for the standard case (be indifferent to swapping), as we showed, and $E(E(B \mid A)-A)$ gets the right answer for the two-stage variant (swap). Is this just a coincidence? Clearly not. They don't get these answers right accidentally, but because the terms of their series reflect something of the causal structure of the problem, in this case how it came about.

If the problem is to be addressed as opposed to abandoned, we need to constrain standard decision theory by the relevant causal structure of the situation. Our solution is to put forward the symmetry of the situation as the significant constraint which relevantly reflects both the evidential structure and the causal structure. (For details, see p. 427.) Our critics are sceptical of this constraint because the argument in favour of the [symmetry] constraint would not convince anyone who did not already agree with their 
conclusions, such as someone who thought that $E(E(B \mid A)-A)$ or $E(B-E(A \mid B))$ was correct

and

The intuition behind their symmetry constraint ... leads directly to the conclusion that swapping and sticking are equipreferable.

So ... [to] go on to apply this intuition to the calculation of average expectations ... adds an unmotivated mathematical epicycle with no explanatory gain. (Measham and Weisberg 2003, p. 000)

Our problem with the first criticism is that (so far as we know) there is no one who thinks that in the standard case either $E(E(B \mid A)-A)$ or $E(B-E(A \mid B))$ gets it right. If they did, what would be their reason for preferring one to the other? After all, unless one thinks the labelling reflects something special about my envelope being mine, the labelling of the envelopes by ' $A$ ' and ' $B$ ' is completely arbitrary. But there is nothing special about my envelope being mine, so by symmetry neither of these two alternative can be preferred over the other. If they don't prefer one to the other, they are committed to the irrationality of thinking it is rational both to swap and not to swap.

Our answer to the second criticism is that the explanatory gain is available only to those who are interested in the problem taken in the way that we have just outlined it, namely, what to do when conditionalizing as a method for evaluating expected utility gives different results depending on how you conditionalize. The challenge is to explain why $E(E(B-A \mid A+B))$ gets the right answer for the standard case and $E(E(B \mid A)-A)$ gets the right answer for the two-stage variant. 
If there were nothing to say in answer to that question then we would agree that showing them to get the right answer achieves nothing on its own. But it turns out there is something to say. Exhibiting the fact that the terms of their series reflect something about the causal structure of the situation suggests a more general truth: that evaluating expected utility by conditionalizing may sometimes require conditionalizing in a way that corresponds to the causal structure on pain (sometimes) of getting the answer wrong.

Calculating expectations by conditionalizing is a vital technique. Many evidential situations do not allow one to compute expectations except via conditionalization. Since economists use such conditionalization, and if they were to do so in a situation allowing of best paradoxical cases, there may be no warning signals that the application of the mathematics is being done in dangerous waters, such as would be given by getting expectations which diverged.

\section{Looking inside your envelope}

We need to distinguish two different cases. The first is the case in which you look in your envelope and calculate a positive expected gain on swapping. The second is the case in which it is argued that since if you looked in your envelope you would calculate a positive expected gain on swapping, you ought to swap anyway (with or without opening the envelope). ${ }^{9}$ We think both of these cases are answered by our remarks in section 4 of our paper.

In the first case the argument seems to be about whether it would in general be a good policy to open your envelope before deciding what to do. Now if

${ }^{9}$ Cf. p. 428 , footnote 11. 
you open your envelope and discover it contains the minimum amount, $£ 1$, then you know you should swap, because the other envelope must contain $£ 2$. So then the question arises, if you do open your envelope, what should you do in all the other cases, when you find more than $£ 1$ in your envelope? We argued that, despite the extra information you have on opening your envelope, whether swapping is better on average is in general unaffected by the knowledge you gain. (See the argument about repeated trials on pp. 429-30.)

But Measham and Weisberg contend that this 'on average heuristic', where the value of $A$ is not fixed, is irrelevant to the peeking case since the value of $A$ is known. Furthermore it is misleading to speak of the $E U$ of repeated trials, since in the peeking case the question is whether or not one should switch in a particular case, given that one has seen a particular amount in envelope $A$. Indeed, with some probability distributions it will be the case that the $E U$ of switching is positive for some values of $A$ and negative for others. (Measham and Weisberg 2003, p. 000)

If there are probability distributions of this type, then, if one of them applies in a two-envelope case in which you look in your envelope (which can be doubted-see footnote 11), it will of course be true that you will know that swapping will be advantageous in some cases but not others, even if the average expected gain, properly calculated, is 0 . (Provided, of course, that you know what the probability distribution is.) Not only would finding $£ 1$ mean you knew you should swap, but perhaps all odd powers of $£ 2$ should be swapped and all even powers kept. The knowledge acquired from looking will 
make all the difference, and the 'average heuristic' will, as they say, be irrelevant. There would be no extra puzzle about looking in such cases.

However, distributions like that described above and others discussed in the literature are not of that type. They are such that if there is an expected gain on swapping in any case where $A>1$, there is an expected gain in every other. In that case the average expected gain must be positive. But if, setting $A=1$ aside, it is 0 , then the expected gain for each case apart from 1 must also be 0 .

In these cases, then, the 'average heuristic' is not irrelevant. Stressing as they do the particular case cannot get us away from the fact that we are considering policies. There are two possible policies: open then swap or be indifferent (unless you find just £1). What does the advice 'open then swap' amount to if not 'on average you will do better if you swap'? But that just insinuates calculating the expected gain on swapping the wrong way again, insinuates calculating $E(E(B \mid A)-A)$ because when you open the envelope you calculate $E(B \mid A=c)-c .^{10}$

We don't deny that it continues to seem plausible to swap because $E(B \mid A=c)-c$ is positive for each $c$, anymore than we deny that in Newcomb's problem one-boxing continues to seem plausible. But it is a misleading plausibility for the reasons we gave in the last section. When the causal structure and evidential structure get out of kilter, there are all sorts of tempting ways, as Lewis so aptly put it, of 'mak[ing] the news ... the news you like best' (Lewis 1981, p. 309). Our suggestion is that the thought to which Measham and Weisberg are appealing is one of those tempting ways. And that, in essence, is our answer to the second case as well. In both cases a

\footnotetext{
${ }^{10}$ See footnote 5 again.
} 
thought is offered which once more persuades you to manage the news rather than managing the world, by calculating $E(E(B \mid A)-A)$ instead of $E(E(B-$ $A \mid A+B)$ ). When instead you constrain your news management by the causal structure, you know that opening the envelope makes no difference to the contents of the envelopes, so cannot make any difference to the correct application of decision theoretic calculations. ${ }^{11}$

So we think there is a significant analogy to be made with Newcomb's problem, although the details of the analogy are not exact. The two-envelope paradox is another case in which evidential structure and causal structure come apart, and resolving the problems encountered by rational decision theory when it appears to follow the evidential structure correctly requires us to bring the causal structure to bear on how rational decision theory is applied.

Department of Philosophy

University of Nottingham

University Park

Nottingham NG7 2RD
MICHAEL CLARK NICHOLAS SHACKEL

$U K$

${ }^{11}$ With this thought in mind, it becomes particularly difficult to see how a probability distribution giving rise to 'the $E U$ of switching [being] positive for some values of $A$ and negative for others' (Measham and Weisberg 2003, p. 000) could ever apply to the two-envelope paradox. As we presented it 'you are presented with two sealed envelopes, one of which contains twice as much money as the other, and you select one at random' (p. 435). Here, the evidence and the causal history would seem to be aligned in ruling out such a distribution. 
michael.clark@nottingham.ac.uk

n.shackel@ntlworld.com

\section{REFERENCES}

Clark, Michael and Shackel, Nicholas 2000: "The Two-Envelope Paradox". Mind 109, pp. 415-442.

Feller, William 1968: An Introduction to Probability Theory and its Applications, Vol. 1, $3^{\text {rd }}$ ed. New York: Wiley.

Jeffrey, Richard C. 1990: The Logic of Decision. 2nd ed. Chicago: University of Chicago Press.

Lewis, David. 1981: “Causal Decision Theory”. Australasian Journal of Philosophy 59, pp. 5-30. Reprinted in Lewis's 1986. Philosophical Papers Vol 2. Oxford: Oxford University Press, 1986 - to which the page reference refers.

Measham, Chris and Weisberg, Jonathan: "Clark and Shackel on the TwoEnvelope Paradox. Mind 112, pp. 000-000.

Ross, Sheldon M. 1988: First Course in Probability Theory. New York: Macmillan Press. 